\title{
Erratum to: Anti-inflammatory activity of the functional groups present in Hippophae rhamnoides (Seabuckthorn) leaf extract
}

Himanshi Tanwar $^{1} \cdot$ Shweta $^{1} \cdot$ Divya Singh $^{1} \cdot$ Shashi Bala Singh ${ }^{1} \cdot$

Lilly Ganju ${ }^{1}$

Published online: 9 May 2017

(C) Springer International Publishing 2017

\section{Erratum to: Inflammopharmacol}

DOI 10.1007/s10787-017-0345-0

Unfortunately, the incorrect author group was published in the original publication. It has been corrected and find below the correct author group for this article.

- Himanshi Tanwar ${ }^{1}$

- Shweta $^{1}$

- Divya Singh ${ }^{1}$

- Shashi Bala Singh ${ }^{1}$

- Lilly Ganju ${ }^{1}$

The original version is also updated.

The online version of the original article can be found under doi:10.1007/s10787-017-0345-0.

Lilly Ganju

lganju@ rediffmail.com

1 Immunomodulation Division, Defence Institute of

Physiology and Allied Sciences, DRDO, Lucknow Road,

Timarpur, Delhi 110054, India 